tissue-engineered heart repair. J Clin Invest. 2013; 123(3):1285-1298.

9. Kim K, et al. Histocompatible embryonic stem cells by parthenogenesis. Science. 2007;315(5811):482-486.

10. Zimmermann WH. Embryonic and embryonic-like stem cells in heart muscle engineering. J Mol Cell Cardiol. 2011;50(2):320-326.

11. Maitra A, et al. Genomic alterations in cultured human embryonic stem cells. Nat Genet. 2005; 37(10):1099-1103.

12. Li TS, Marban E. Physiological levels of reactive oxygen species are required to maintain genomic stabil- ity in stem cells. Stem Cells. 2010;28(7):1178-1185.

13. Figueiredo C, Horn PA, Blasczyk R, Seltsam A. Regulating MHC expression for cellular therapeutics. Transfusion. 2007;47(1):18-27.

14. Rao MS, Auerbach JM. Estimating human embryonic stem-cell numbers. Lancet. 2006;367(9511):650.

15. Liao SY, et al. Proarrhythmic risk of embryonic stem cell-derived cardiomyocyte transplantation in infarcted myocardium. Heart Rhythm. 2010;7(12):1852-1859.

16. Roell W, et al. Engraftment of connexin 43-expressing cells prevents post-infarct arrhythmia. Nature. 2007;450(7171):819-824
17. Shiba Y, et al. Human ES-cell-derived cardiomyocytes electrically couple and suppress arrhythmias in injured hearts. Nature. 2012;489(7415):322-325.

18. Segers VF, Lee RT. Biomaterials to enhance stem cell function in the heart. Circ Res. 2011;109(8):910-922.

19. Zimmermann WH, et al. Engineered heart tissue grafts improve systolic and diastolic function in infarcted rat hearts. Nat Med. 2006;12(4):452-458.

20. Le Blanc K, et al. Mesenchymal stem cells for treatment of steroid-resistant, severe, acute graftversus-host disease: a phase II study. Lancet. 2008; 371(9624):1579-1586

\title{
Bathing the brain
}

Warren J. Strittmatter

Departments of Medicine (Neurology) and Neurobiology, Duke University Medical Center, Durham, North Carolina, USA.

\begin{abstract}
The brain and spinal cord are surrounded by cerebrospinal fluid, which provides a mechanically stable environment for these delicate structures against the forces of gravity and sudden acceleration and deceleration. Neurons and glia comprising the parenchyma of the brain are enveloped in their microenvironment by interstitial fluid. Interstitial fluid has long been considered to be unaffected by the production and flow of cerebrospinal fluid outside the brain parenchyma. However, two recent papers by Iliff et al. demonstrate that cerebrospinal fluid enters the deep substance of the brain, mixes with the interstitial fluid surrounding neurons and glia, and plays an important role in the exchange and clearance of molecules in the interstitial space of the central nervous system.
\end{abstract}

Hippocrates observed that the brain is suspended in a fluid, but the functions of that cerebrospinal fluid (CSF) vital to brain function have only been slowly revealed. Francois Magendie compared the brain suspended in CSF to the fetus suspended in amniotic fluid, and indeed, CSF does provide mechanical support for the brain. Since the brain is neutrally buoyant in this environment, CSF provides protection of neural tissue from the force of gravity and from sudden changes in velocity during head impact. The production of CSF by the choroid plexus in the lateral ventricles, its transit through the third and fourth ventricles, its coursing over the exterior surface of the brain and spinal cord, and its ultimate uptake at the arachnoid granulations in the large draining veins was not fully described until the early 1900s (Figure 1A and ref. 1).

\section{Into the soup}

Virtually every cell throughout the body is surrounded by interstitial fluid, which

Conflict of interest: The author has declared that no conflict of interest exists.

Citation for this article: J Clin Invest. doi:10.1172/ JCI68241. plays critical roles in cellular homeostasis, including maintaining osmotic pressure, supplying nutrients, and equilibrating the concentrations of molecules by diffusion. In tissues throughout the body, interstitial fluid is slowly exchanged by intermixing with lymph that transits unidirectionally through the tissue to draining lymphatic vessels, first identified anatomically in the 1600s. The convectional flow of lymph mixes with interstitial fluid and facilitates the transit of molecules and cells within the tissue. Brain, however, does not contain such discrete lymphatic vasculature. The hypothesis that brain possesses a "lymphatic" system with the same physiologic functions, but with different anatomy than that found throughout the remainder of the body, was initially proposed following the observations that CSF is rapidly removed from the neonatal brain, even though the venous system in the neonate contains only few arachnoid granulations, and, additionally, that CSF flows abundantly and unidirectionally along the cribriform plate in the nose, which contains no arachnoid granulations. Injection of radiolabeled albumin into the CSF revealed that half of the albumin tracer eventually drains from the cribriform plate into the cervical lymphatic system $(2,3)$. CSF also drains into the lymphatic system through the sheaths of cranial and spinal nerves (4).

\section{Mapping the flow}

Recently, Iliff et al. demonstrated that the directional flow of CSF through the parenchyma of the brain mixes with brain interstitial fluid and facilitates its clearance (5). In this issue of JCI, this group used nuclear MRI to visualize and quantify the pathway in the intact organism, a critical advance in translating this knowledge to the clinic (6).

Iliff et al. injected a fluorescent tracer into the CSF at different sites and initially observed that tracer injected into the lateral ventricles, the site of CSF production, minimally diffused into the parenchyma of the brain, while in contrast, tracer injected into the cisternal space surrounding the external surface of the brain entered the brain parenchyma. Two-photon laser scanning microscopy revealed that fluorescent dye injected into the cisternal space entered the deep brain parenchyma by traveling along the outside of cerebral arteries, entering the brain via the para-arterial channels of the Virchow-Robin space (6). Once deep into the brain, dye diffused into the parenchyma, bathing neurons and glia and mixing with the interstitial fluid. Within the parenchyma, CSF mixed with interstitial fluid, subsequently traveled along the external surface of large caliber veins, and reentered either the subarachnoid space or drained into the cervical lymphatic system (Figure 1B). This demonstration that CSF robustly transits through the parenchyma of the brain and mixes with interstitial fluid has important implications for 
A

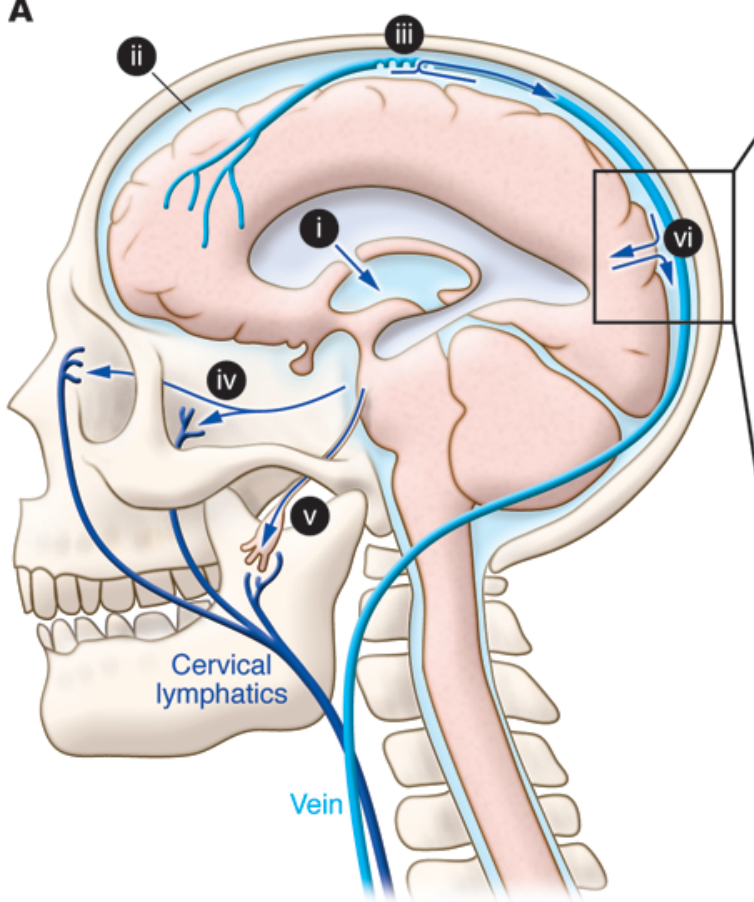

B

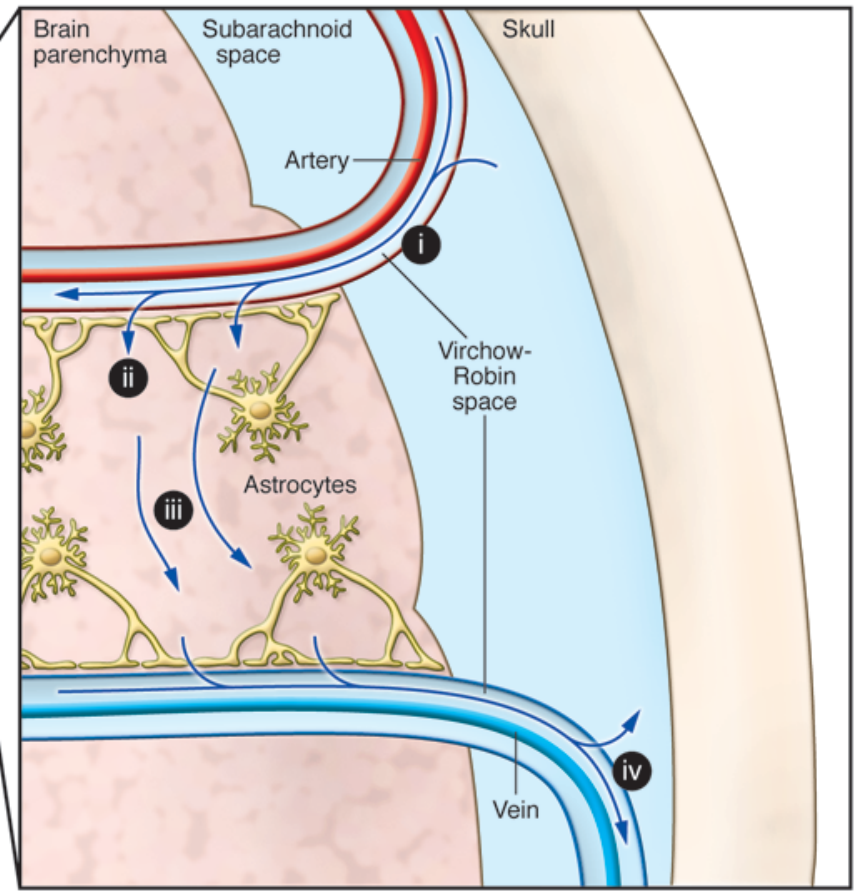

\section{Figure 1}

CSF and its traffic to the lymphatic system. (A) Formation and major egress pathways of CSF. CSF is produced primarily at the choroid plexus of the lateral ventricles (i) and transits through the ventricles of the brain to eventually enter the subarachnoid space surrounding the brain and spinal cord (ii). The primary route of egress of CSF occurs at the arachnoid granulations of large draining veins (iii). CSF also drains into the cervical lymphatic system through the cribriform plate (iv) and through nerve sheaths (v). CSF enters the interstitial fluid compartment in brain parenchyma via the paravascular pathway (vi). This figure was published in: Sakka L, Coll G, Chazal J. Anatomy and physiology of cerebrospinal fluid. European Annals of Otorhinolaryngology, Head and Neck Diseases 2011;128(6):309:316. Copyright (C) 2013 Elsevier Masson SAS. All rights reserved (4). (B) The paravascular system. CSF enters brain parenchyma along the Virchow-Robin space surrounding large penetrating arteries (i). CSF then transits around, or through, the end feet of astrocytes that largely surround the microvasculature (ii). CSF then mixes with and transports interstitial fluid (iii), which then enters the Virchow-Robin space surrounding exiting veins and reenters the subarachnoid space or drains to cervical lymphatics (iv). Modified with permission from Science Translational Medicine (5).

understanding the mechanisms of brain homeostasis, both in normal function and in disease. The investigators term this flow of CSF from the para-arterial space, its admixing with interstitial fluid, and its exit from the brain parenchyma through the para-venous space the "paravascular pathway" or "glymphatic system."

The large external brain arteries, such as the basilar artery, provide the major sites of penetration of CSF into the deep brain parenchyma. The primary pathways for entry of CSF into brain parenchyma occur at the pituitary recess, the olfactory bulb, and in the pineal recess. This initial penetration of CSF along these para-arterial channels appears to involve bulk flow, since molecules of vastly different molecular weight are transported at similar rates. Movement of CSF from the para-arterial space into the interstitial space of the brain occurs either through or around the end feet of astrocytes that almost totally surround the microvasculature of the brain (ref. 6 and Figure 1B). At this anatomic site, the diffusion of CSF into the interstitial fluid is determined in part by the molecular weight of molecules in the CSF, with diffusion of small molecular weight species occurring markedly faster than that of large molecular weight species. The unidirectional flow of CSF from the para-arterial space into brain parenchyma is facilitated in part by water transport within the astrocyte through the water channel aquaporin-4 (5), which localizes primarily in the astrocytic end feet surrounding the vasculature.

\section{Translating to clinical utility}

The mechanism and the rate at which various molecular species are removed from the parenchyma of the brain by this paravascular pathway may be relevant to the pathology of a number of neurological conditions. For example, the amyloid A- $\beta$ peptide, implicated in Alzheimer's disease pathogenesis, is released by neurons into the interstitial space. The subsequent trafficking and accumulation of A- $\beta$ in the brain interstitial space, either as soluble oligomers or as insoluble amyloid, appears critical in the mechanism of this disease (7). In a recent article, Iliff et al. demonstrated that the paravascular pathway facilitates the clearance of this peptide (5). Identifying the anatomic sites of A- $\beta$ clearance, and its rate of clearance, may eventually provide the earliest detectable abnormality preceding the clinically detectable decline of cognition in this disease. The accumulation of A- $\beta$ amyloid around arteries commonly observed in Alzheimer's disease may retard the rate of paravascular clearance of this or other molecules, thereby accelerating disease progression. Indeed, researchers recently linked variants of the Trem2 gene, an immune receptor that mediates phagocytosis and may be involved in the 
clearance of amyloid, with increased risk of developing Alzheimer's disease (8). Other neurodegenerative diseases also result from aberrant protein accumulation in the interstitial space of the brain. Prion diseases, such as Creutzfeld-Jacob disease, Parkinson's disease, and frontotemporal dementia, may result from cell-to-cell transmission of protein in the extracellular, interstitial space (9). Change in the kinetics of clearance of such proteins may be important in determining the rate of progression of these neurodegenerative disorders. Furthermore, the concentration and the distribution of molecules in the interstitial space of the brain are critical not only in chronic neurodegenerative disorders. Accumulation of interstitial fluid following mechanical and metabolic insults produces brain edema and neurologic symptoms, including coma and death. Using contrast-enhanced MRI to assess the kinetics of paravascular CSF flow may give important information about the impending appearance, and clinical progression, of this clinically important complication.

Address correspondence to: Warren J. Strittmatter, Duke University Medical Center, Bryan Research Bldg., Room 227, Durham, North Carolina 27710, USA. Phone: 919.684.0053; Fax: 919.681.7198; E-mail: warren@neuro.duke.edu.

1. Hajdu SI. A note from history: Discovery of the cerebrospinal fluid. Annals of Clin and Lab Sci. 2003;33(3):334-336.

2. Boulton M, et al. Drainage of CSF through lymphatic pathways and arachnoid villi in sheep: Mea- surement of ${ }^{125} \mathrm{I}$-albumin clearance. Neuropathol Appl Neurobiol. 1996;22(4):325-333.

3. Johnston M, et al. Evidence of connections between cerebrospinal fluid and nasal lymphatic vessels in humans, non-human primates and other mammalian species. Cerebrospinal Fluid Res. 2004;1(1):2.

4. Sakka L, Coll G, Chazal J. Anatomy and physiology of cerebrospinal fluid. Eur Ann Otorbinolaryngol Head Neck Dis. 2011;128(6):309-316.

5. Iliff JJ, et al. A paravascular pathway facilitates CSF flow through the brain parenchyma and the clearance of interstitial solutes, including amyloid b. Sci Transl Med. 2012;4(147):147ra111.

6. Iliff JJ, et al. Brain-wide pathway for waste clearance captured by contrast-enhanced MRI. J Clin Invest. 2013;123(3):1299-1309.

7. Huang Y, Mucke L. Alzheimer mechanisms and therapeutic strategies. Cell. 2012;148(6):1204-1222.

8. Jonsson T, et al. Variant of TREM2 associated with the risk of Alzheimer's disease. N Engl J Med. 2013; 368(2):107-116.

9. Frost B, Jacks RL, Diamond MI. Propagation of tau misfolding from the outside to the inside of a cell. J Biol Chem. 2009;284(19):12845-12852.

\title{
Calcium flux and endothelial dysfunction during acute lung injury: a STIMulating target for therapy
}

\author{
Eric J. Seeley, ${ }^{1}$ Paul Rosenberg, ${ }^{2}$ and Michael A. Matthay ${ }^{1}$
}

\begin{abstract}
${ }^{1}$ Cardiovascular Research Institute and Departments of Medicine and Anesthesia, UCSF, San Francisco, California, USA. ${ }^{2}$ Department of Medicine,
\end{abstract} Duke University, Durham, North Carolina, USA.

\begin{abstract}
Bacterial pathogen-associated molecular pattern molecules (PAMPs) such as LPS activate the endothelium and can lead to lung injury, but the signaling pathways mediating endothelial injury remain incompletely understood. In a recent issue of the JCI, Gandhirajan et al. identify STIM1, an ER calcium sensor, as a key link between LPS-induced ROS, calcium oscillations, and endothelial cell (EC) dysfunction. In addition, they report that BTP2, an inhibitor of calcium channels, attenuates lung injury. This study identifies a novel endothelial signaling pathway that could be a future target for the treatment of lung injury.
\end{abstract}

During infection, circulating bacterial products and endogenous cytokines stimulate the endothelium, setting off a cascade of vascular activation, including the increased expression of vascular adhesion molecules and regional increases in endothelial permeability. This response, the result of millennia of warfare between mammals and microbes, is beneficial during compartmentalized infections such as those of soft tissue and pneumonia. The alterations in vascular adhesion and permeability enable neutrophils and

Conflict of interest: The authors have declared that no conflict of interest exists.

Citation for this article: J Clin Invest. doi:10.1172/ JCI68093. monocytes to penetrate infected tissues, where they sequester and kill pathogens. However, similar physiological responses in vulnerable vascular beds, such as the capillary networks of the lungs and kidneys, can lead to multiple organ failure in critically ill patients. Achieving a deeper understanding of the signals that regulate vascular integrity during host defense and organ injury would be an important step in reducing vascular injury during human sepsis.

\section{The endothelium during sepsis and lung injury}

A major focus of recent research is the function of TLR4 within the vascular endothelium, where, through an inter- action with LPS, it plays a critical role as both protector and protagonist during sepsis and multiple organ failure. Mice that express TLR4 exclusively on ECs can detect and clear intraperitoneal E. coli infection as rapidly as wild-type counterparts (1). Thus, the endothelium, without the help of TLR4-expressing immune cells, can sense and eradicate intravascular infection. In addition to its role in immune surveillance and activation, the signaling pathways downstream of endothelial TLR4 are critical to the pathogenesis of organ injury, as illustrated by a mouse model in which a degradation-resistant form of $\mathrm{I} \mathrm{B}$, the cytoplasmic inhibitor of NF- $\kappa \mathrm{B}$, is expressed in ECs (2). These mice maintain the ability to sense and clear pathogens, yet have decreased organ injury and improved survival during LPS- or E. coli-induced peritonitis $(2,3)$. Thus, the endothelium is both a sensor of infection and a mediator of septic organ injury. Understanding how perturbations in TLR4 signaling lead to endothelial dysfunction would be a major step toward treating vascular dysfunction during sepsis. 